\title{
Actividad física, condición física y autoconcepto en escolares de 8 a 12 años Physical activity, physical fitness and self-concept in schoolchildren aged between 8 to 12 years old Andres Rosa Guillamón, Eliseo Garcia Canto, Pedro Jose Carrillo López Universidad de Murcia (España)
}

Resumen.Este artículo analizó la relación entre actividad física, condición física y autoconcepto. Un total de 103 escolares (8-12 años) participaron en la evaluación de dichas variables. La condición física se valoró mediante diversas pruebas de la Batería ALPHA-Fitness, el nivel de actividad física se calculó a través del cuestionario PACE y el autoconcepto se midió utilizando la Escala de Autoconcepto de Piers-Harris. Las pruebas estadísticas fueron: T-student, U de Mann-Whitney y Krustal-Wallis. Aquellos físicamente activos tuvieron mejores registros en las dimensiones conductual, intelectual, falta de ansiedad y global. Aquellos con mayor condición física mostraron un mejor autoconcepto físico $(p=.013)$, social $(p=.003)$ y global $(p=.085)$. Aquellos físicamente activos y con mayor condición física tuvieron un mejor autoconcepto intelectual $(p=.007)$, social $(p=.010)$ y global $(p=.010)$. Ser físicamente activo y tener un mayor nivel de condición física podría predecir un autoconcepto más positivo en escolares de la Región de Murcia. Palabras clave: Escolares, salud, condición física, ejercicio físico, autoconcepto.

Abstract. This article analized the relationship between physical activity, physical condition and self-concept. A total of 103 schoolchildren (8-12 years old) participated in the assessment of these variables. Physical fitness was assessed through various tests from the ALPHA-Fitness Battery, level of physical activity was calculated through the PACE questionnaire, and self-concept was measured using the Piers-Harris Self-concept Scale. T-student, Mann-Whitney U, and Krustal-Wallis were employed for statistical analysis. Those who were physically active had better values in behavioral and intellectual domains of self-concept, as well as in lack of anxiety, and global self-concept. Those with greater physical fitness showed a better physical ( $\mathrm{p}=.013)$, social $(p=.003)$ and global $(p=.085)$ self-concept. Those physically active with greater physical fitness had a better intellectual ( $p=.007)$, social $(p=.010)$ and global $(p=.010)$ self-concept. Being physically active and having a higher level of fitness could predict a more positive selfconcept in schoolchildren of Murcia (Spain).

Keywords: Schoolchildren, health, physical exercise, physical fitness, self-concept.

\section{Introducción}

La percepción que cada persona tiene de sí misma condiciona la interpretación del mundo que la rodea, estableciéndose una relación entre su autoconcepto y su conducta intencional (Bufford, 1986). El autoconcepto se define como la autopercepción de una persona en relación a aspectos que considera trascendentales para su vida (Piers \& Herzberg, 2002). Constituye un marco de referencia desde el que interpretar la realidad externa y las propias experiencias, influye en el rendimiento en distintos ámbitos, condiciona las expectativas y las emociones, contribuye a la salud y al equilibrio psíquico(García, 2013; ZagalazSánchez, Castro-López, Valdivida-Moral, \& Cachón-Zagalaz, 2018) y da significado a la existencia personal (Flores, Medrano \& Victoria, 2014).

En la evolución del autoconcepto, la preadolescencia se presenta como una de las etapas más importantes debido a las profundas transformaciones en funciones y estructuras (corporales, cerebrales, sexuales, emocionales y sociales) que pueden determinar la construcción equilibrada de símismo (Mendo-Lázaro, Polo-del-Río, Amado-Alonso, Iglesias-Gallego \& León-del-Barco, 2017).

La conformación positiva del autoconcepto depende también de los núcleos socioculturales que rodean a la persona (Moreno, Cervelló \& Moreno, 2008) y de un desarrollo óptimo de las distintas dimensiones que lo conforman (Rodríguez-García, Tárraga, Rosa, García-Cantó, Pérez-Soto, Gálvez, A. \& Tárraga, 2014; Gálvez, Rodríguez-García, Rosa, García-Cantó, Pérez-Soto, Tárraga \& Tárraga, 2016). Estas dimensiones o dominios varían en número e identidad según el modelo de autoconcepto que se adopte; entre las dimensiones más aceptadas en la literatura científica se encuentran la académica, la emocional, la física, la social y la familiar (Shavelson, Hubner \& Stanton, 1976). Autores como Piers \& Herzberg (2002) añaden a estas, la conductual. Cada una de estas dimensiones están compuestas, a su vez, por subdominios que especifican la percepción de la persona en cada faceta de su vida (Zabala \& Palacios, 2008).

En la investigación educativa, la importancia concedida al autoconcepto multidimensional responde a la presunción de que no se

Fecha recepción: 30-03-18. Fecha de aceptación: 21-09-18

Eliseo Garcia Canto

eliseo.garcia@um.es puede entender el rendimiento y la conducta de los niños sin considerar las percepciones que el sujeto tiene de sí mismo y, en particular, de su propia competencia (Goñi \& Fernández, 2008).

Es en la etapa educativa, cuando se llevan a cabo las primeras tendencias a determinadas conductas, concentrándose luego en intereses que durante la adolescencia se pueden convertir en categorías de valor decisivas para la vida futura (Guillen \& Ramírez, 2011). Así sucede, entre otros, con los patrones de actividad física (AF) habitual. La realización de $\mathrm{AF}$ desde edades tempranas puede determinar, en buena parte, la configuración de hábitos de AF saludable y el mantenimiento de una condición física(CF) adecuada a lo largo de la propia vida (Rosa, García-Cantó, Rodríguez-García, Pérez-Soto \& Tárraga-Marcos, Tárraga-López, 2017; Gallego, Sánchez, Vacas \& Zagalaz, 2016). La consolidación de un estilo de vida activo y saludable basado en la realización de $\mathrm{AF}$ es una de las principales estrategias frente a las posibles alteraciones del autoconcepto de los sujetos jóvenes (Gálvez et al., 2016; Reigal, Videra, Parra \& Juárez, 2012).

La realización de AF bajo unos determinados parámetros de frecuencia, intensidad y duración puede contribuir a la preservación del bienestar emocional de los niños (Gálvez, Rodríguez-García, GarcíaCantó, Rosa, Pérez-Soto, Tárraga-Marcos \& Tárraga-Marcos, 2015a), siendo el autoconcepto multidimensional uno de los principales constructos en las personas que se encuentran construyendo las bases de su personalidad(Sonströem, 1998). En este sentido, las conclusiones de la aplicación de diversas intervenciones en el ámbito educativo muestran que mejorar las creencias de los niños sobre su capacidad para el ejercicio puede aumentar la motivación para estar físicamente activos (Strauss, Rodzilsky, Burack \& Colin, 2001), ya que demuestran una mayor predisposición a disfrutar y mantener el interés hacia la AF generando conductas de adherencia (Weiss, 2000). Estos hallazgos sugieren, asimismo, que la mejora del estado de CF a través de la AF habitual podría tener una influencia positiva no solo en la salud física (Casajus, Ortega, Vicente-Rodriguez, Leiva, Moreno \& Ara, 2012) sino también en la salud mental, a partir de los posibles efectos sobre el autoconcepto (Rodríguez-García, Gálvez, García-Cantó, Pérez-Soto, Rosa, Tárraga \& Tárraga, 2015; Sonströem, Harlow \& Josephs, 1994) y la calidad devida percibida (Rosa-Guillamón, García-Cantó, RodríguezGarcía \& Pérez-Soto, 2016).

Recientes investigaciones indican que aquellos jóvenes físicamente activos (García-Cantó \& García-López, 2011; Kyle, Hernández-Mendo, Reigal-Garrido \& Morales-Sánchez, 2016; Moore, Mitchell, Bibeau \& 
Bartholomew, 2011; Moreno, Cervelló \& Moreno, 2008; Petty, Davis, Tkacz, Young-Hyman \& Waller, 2009; Planinšec \& Fošnariè, 2005) o que tienen un mejor estado de CF (Gálvez et al., 2016; Mayorga, Viciana \& Cocca, 2012; Rodríguez-García et al., 2014; Rodríguez-García et al., 2015; Sánchez-Alcaraz \& Gómez-Mármol, 2014; Vedul Kjelsås, Sigmundsson, Stensdotter \& Haga, 2012) presentan un mejor autoconcepto.

Por el contrario, en la literatura científica se encuentran estudios donde se pone en duda la relación entre la AF, la CF y el autoconcepto, concluyendo que la asociación entre estas variables es débil aludiendo, principalmente, a la temprana edad de los sujetos estudiados (Guillén \& Ramírez, 2011), o que esta relación se reduce a dimensiones concretas, especialmente la emocional y la física (García-Sánchez, BurgueñoMenjibar, López-Blanco \& Ortega, 2013; Reigal-Garrido, BecerraFernández, Hernández-Mendo \& Martín-Tamayo, 2014), sugiriendo que los efectos de la práctica deportiva y la mejora de la CF sobre el mismo se encuentran sobredimensionados.

A pesar de los argumentos que apoyan la relación entre la AF como conducta intencional positiva para un estilo de vida saludable (MartínezBaena, Mayorga-Vega \& Viciana, 2018; Rosa, García-Cantó, RodríguezGarcía, Pérez-Soto, Tárraga-Marcos, \& Tárraga-López, 2017), la CF como biomarcador de salud física (Ruiz et al., 2011) y mental (RosaGuillamón \& García-Cantó, 2016) y el autoconcepto como constructo fundamental en la conformación equilibrada de la personalidad humana (Madariaga \& Goñi, 2009), diversos aspectos quedan por ser aclarados en el análisis del autoconcepto multidimensional en la edad escolar. Van den Berghe, Vanstenkiste, Cardon, Kirk \& Haerens (2014) advierten que esnecesario seguir investigando en estas edades ya que, al poder concretar las relaciones entre la AF y el autoconcepto multidimensional se pueden establecer las intervenciones educativas efectivas. En base a esto, el objetivo de este trabajo fue analizar la relación entre la AF, la CF y el autoconcepto multidimensional en una muestra de escolares de educación primaria del sureste español.

\section{Material y método}

\section{Participantes}

Estudio empírico de tipo descriptivo, relacional y de corte transversal (Thomas \& Nelson, 2007) realizado con 103 escolares, entre ocho y 12 años (media \pm desviación estándar $=9.94 \pm 1.40$ años). Los escolares pertenecían a dos centros públicos de educación primaria de la Región de Murcia (España) y fueron seleccionados mediante muestreo no probabilístico intencional (véase Tabla 1). Se plantearon los siguientes criterios de exclusión: 1) padecer patologías osteo-articulares agudas o crónicas; 2) tener una enfermedad mental; y, 3) no presentar por escrito consentimiento informado de los padres o tutores legales.

La investigación se llevó a cabo de acuerdo con las normas deontológicas reconocidas por la Declaración de Helsinki (revisión de 2013). Este trabajo pertenece a un estudio más amplio que forma parte de una tesis doctoral desarrollada en el Departamento de Expresión Plástica, Musical y Dinámica de la Universidad de Murcia (Murcia, España).

\begin{tabular}{|c|c|c|}
\hline & Varones $(\mathrm{n}=40)$ & Mujeres $(n=63)$ \\
\hline 8 años, $n$ (\%) & $10(45.5)$ & $12(54.5)$ \\
\hline 9 años, $n$ (\%) & $4(21.1)$ & $15(78.9)$ \\
\hline 10 años, $n$ (\%) & $9(37.5)$ & $15(62.5)$ \\
\hline 11 años, $n(\%)$ & $9(47.4)$ & $10(52.6)$ \\
\hline 12 años, $n(\%)$ & $8(42.1)$ & $11(57.9)$ \\
\hline
\end{tabular}

\section{Variables e instrumentos}

La valoración previa de los participantes para realizar los test de CF se realizó a través del Cuestionario de Aptitud para la Actividad Física (PAR-Q) (Thomas, Reading \& Shepard, 1992). La finalidad de este instrumento fue contribuir a la selección preliminar segura de sujetos para participar en estetrabajo en el cual se emplean test de campo de medición de la CF. Este instrumento ha sido empleado en investigaciones previas con sujetos de ocho a 12 años (Gálvez etal., 2016; Rodríguez-
García et al., 2014; Rosa-Guillamón et al., 2016).

Para medir la salud relacionada con la CF se seleccionaron diversas pruebas de la Batería ALPHA-Fitness basada en la evidencia (Ruiz et al., 2011). Se realizaron modificaciones permitidas en el Manual de Instrucciones de la Batería ALPHA-Fitness (2016). No se midieron los pliegues cutáneos por motivos de tiempo limitado; se añadió el test de carrera 4 x 10 m de la versión extendida (Ruiz et al., 2011). La fiabilidad y la validez de estos test se encuentran bien establecidas en sujetos de ocho a 12 años (Gálvez et al., 2015; Rosa et al., 2016).Los parámetros que se evaluaron fueron los siguientes.

Capacidad motora. La velocidad-agilidad fue medida con el test de carrera 4 x 10 m (Vicente-Rodríguez et al., 2012). Se utilizó como instrumento de medida un cronómetro profesional (HS-80TW-1EF, Casio, Tokio, Japan).

Capacidad músculo-esquelética. La fuerza de prensión manual se midió con un test de dinamometría manual empleando un dinamómetro digital con agarre ajustable (TKK 5041 Grip D, Takei, Tokio, Japan). Se utilizó una regla-tabla para adaptar la amplitud del agarre (EspañaRomero et al., 2010). La potencia explosiva del tren inferior se valoró mediante el test de salto longitudinal a pies juntos (Castro-Pinero, Ortega, Artero, Girela-Rejón, Mora, Sjostrom \& Ruiz, 2010). Se empleó para su medida una cinta métrica de PVC y fibra de vidrio (Modelo 74Y100M, CST/Berger, Chicago, USA).

Capacidad aeróbica. Se empleó el test de Course-Navette (Lèger, Mercier, Gadoury \& Lambert 1988) para medir la capacidad aeróbica. Se utilizó un equipo audio portátil (Behringer EPA40, Thomann, Burgebrach, Germany) y un dispositivo de memoria USB (Hayabusa, Toshiba, Tokio, Japan).

Las variables de CF evaluadas (velocidad-agilidad, fuerza deprensión manual, potencia explosiva del tren inferior y capacidad aeróbica) fueron transformadas dividiendo cada uno de los valores observados por el valor máximo de dicha variable. Se sumaron los valores de fuerza de prensión manual, potencia explosiva del tren inferior y capacidad aeróbica y se restó el valor de velocidad-agilidad, dando como resultado una variable de CF global denominada zCF-ALPHA (Rosa-Guillamón \& García-Cantó, 2016). Se categorizó a los participantes con menor CF (mzCF-ALPHA) cuando el valor fue $\mathrm{X}<\mathrm{P}_{50}$; $\mathrm{y}$ mayor CF (MzCFALPHA) cuando el valor fue $X$ e» $P_{50^{\circ}}$.

Para medir el nivel deAF se utilizó el cuestionario Physician-based Assessment and Counseling for Exercise (PACE; Prochaska, Sallis \& Long, 2001). Este cuestionario fue desarrollado para valorar el nivel de AF de los participantes del estudio Physician-based Assessment and Counseling for Exercise. El estudio PACE, iniciado en 1990, tenía como propósito desarrollar instrumentos para evaluar el nivel de AF habitual de niños y adolescentes, así como el desarrollo de protocolos de asesoramiento para llevar un estilo de vida más activo y saludable (Calfas et al., 1996; Patrick et al., 2001). Este cuestionario valora con dos ítems, 1) ¿Cuántos días en la última semana? (PACE 1); y, 2) ¿cuántos días en una semana habitual?(PACE 2), el nivel de AF realizada por el individuo durante un mínimo de 60 minutos.

En el estudio de validez con 138 sujetos jóvenes, el cuestionario mostró que el resultado compuesto obtenido de ambas preguntas ([PACE 1+PACE 2]/2) lograba una correlación $r=0.40(r=0.42$ y $r=0.32$ para varones y mujeres, respectivamente) frente a las medidas objetivas obtenidas mediante un acelerómetro CSA; así como una fiabilidad testretest evaluada por el coeficiente de correlación intraclase(CCI) de 0.77 . En este trabajo, el cuestionario mostró una buena fiabilidad medida a través de la prueba Alpha de Cronbach (á = 0.847).

Empleando los resultados de este test, se categorizó a los participantes según su nivel de AF: no activos ( $\mathrm{X}<5$ horas) y activos (X e» 5 horas), tal y como se establece en la literatura científica (Department of Health, 2004; Prochaska et al. 2001).Se creó también una nueva variable combinando los niveles de $\mathrm{AF}$ y $\mathrm{CF}$, clasificando a los participantes en cuatro grupos denominados: $\mathrm{A}=$ no activo $/ \mathrm{mzCF}-\mathrm{ALPHA}$; $\mathrm{B}$ =no activo/MzCF-ALPHA; C =activo/ mzCF-ALPHA; y D = activo/ MzCF-ALPHA

Para evaluar el autoconcepto se utilizó la Escala de Autoconcepto 
de Piers-Harris (Piers y Herzberg, 2002), elaborada para ser administrada a sujetos de 7 a 12 años y adaptada al castellano por Cardenal y Fierro (2003). Se trata de un instrumento ampliamente utilizado en el ámbito educativo (Gálvez et al., 2015; Gálvez et al., 2016; RodríguezGarcía et al., 2014; Rodríguez-García et al., 2015), con una metodología de cumplimentación basada en ítems de tipo dicotómico (sí/no), que evalúan el grado de acuerdo o desacuerdo con lo enunciado en cada ítem. El instrumento está compuesto por diversas sub-escalas: conductual, intelectual, física, falta de ansiedad, social o popularidad, felicidad subjetiva-satisfacción vital y global. Un mayor autoconcepto se relaciona con puntuaciones elevadas en las distintas dimensiones, excepto para la sub-escala de falta de ansiedad, en la que puntuaciones superiores describen menores niveles de ansiedad.

Las propiedades psicométricas de este instrumento han sido documentadas en otros estudios que muestran una adecuada consistencia interna y una alta fiabilidad y validez (Gálvez, Rodríguez-García, Rosa, García-Cantó, Pérez-Soto, Tárraga \& Tárraga, 2015c; Gálvez et al., 2016; Rodríguez-García et al., 2014; Rodríguez-García et al., 2015). En esta investigación se han aplicado pruebas de fiabilidad de los ítems de la escala y un análisis factorial confirmatorio para verificar la agrupación de los ítems en las distintas sub-escalas definidas originalmente. Todos los ítems de la escala poseen consistencia y fiabilidad, ya que de ser eliminado alguno de ellos disminuye sensiblemente la varianza explicada, y la fiabilidad global en cada sub-constructo y en la escala global. La escala mostró una buena fiabilidad medida a través de la prueba Alpha de Cronbach (á= 0.892); la fiabilidad de los diferentes sub-constructos fue la siguiente: conductual (á = 0.921), intelectual (0.935), físico (á = 0.845), falta de ansiedad (á $=0.901$ ), social o popularidad (á $=0.856$ ) y felicidad subjetiva-satisfacción vital (á=0.845).

El análisis factorial confirmatorio con rotación varimax confirmó las seis subescalas en que queda estructurado dicho instrumento. Los subconstructos y la escala global explican una varianza que ofrece consistencia al instrumento aplicado. Los porcentajes de varianza explicada en los diferentes subconstructos fueron los siguientes: conductual (15.11\%), intelectual (14.89\%), físico (13.98\%), falta de ansiedad (13.06\%), social o popularidad (11.65\%) y felicidad-satisfacción con la vida $(0.94 \%)$. La varianza total explicada fue del $78.66 \%$. La escala utilizada en la presente investigación es un instrumento válido y fiable para evaluar el autoconcepto de los sujetos integrantes de la muestra.

\section{Procedimiento}

Este estudio fue realizado durante el curso académico (2016/17). Se recibió por escrito la autorización para la realización del estudio. El explorador colaborador era graduado en ciencias del deporte. El proceso de recogida de datos se desarrolló durante el mes de octubre. La administración de las pruebas se realizó en horario lectivo. Se recomendó a los padres de los escolares que no realizasen AF la tarde anterior, no variasen su alimentación habitual y vistiesen ropa deportiva ligera. La administración de las pruebas se hizo por grupo clase realizando una demostración previa de cada prueba, dejando intervalos de 5-10 minutos entre medidas. Se realizó un calentamiento estándar. La Escala de Autoconcepto de Piers Harris se administró en un aula que permitía suficiente para mantener la privacidad en la cumplimentación, con la presencia del explorador para asegurar que se cumplimentaban adecuadamente. La duración media en la cumplimentación fue de 20 minutos.

\section{Análisis estadístico}

La distribución de la muestra resultó no normal al aplicar la prueba de Kolmogorov-Smirnov. Las diferencias según sexo (varones $=0$ y mujeres $=1$ ) se analizaron mediante la prueba U de Mann-Whitney para variables continuas y el test de chi-cuadrado de Pearson para las variables categóricas. Se realizó un análisis mediante la prueba U de Mann-Whitney para estudiar la relación entre AF y autoconcepto y entre CF y autoconcepto. Se realizó una prueba $\mathrm{H}$ de Kruskal-Wallis para analizar la asociación entre la AF, la CF y el autoconcepto. El análisis estadístico fue realizado con el programa SPSS (23.0v., Chicago, Illinois, EE. UU.). La significancia estadística se fijó a un valor $p$ d» .05.

\section{Resultados}

La tabla 2 muestra los valores promedio de las puntuaciones directas en los test de CF, así como en las seis dimensiones del autoconcepto y el autoconcepto global. Se presenta la distribución de la muestra en función del nivel de AF establecido en el estudio PACE (no activo y activo), el nivel de CF (zmCF-ALPHA y zMCF-ALPHA) y la relación combinada entre AF y CF (no activo/ zmCF-ALPHA y no activo/ zMCF-ALPHA; activo/zmCF-ALPHA y activo/zMCF-ALPHA).

\begin{tabular}{|c|c|c|}
\hline & Varones $(\mathrm{n}=40)$ & Mujeres $(\mathrm{n}=63)$ \\
\hline $4 \times 10 \mathrm{~m}(\mathrm{~s})$ & $13.4 \pm 1,1$ & $14.1 \pm 1.2$ \\
\hline Dinamometría manual(kg) & $31.1 \pm 9.0$ & $27.4 \pm 8.4$ \\
\hline Salto longitudinal $(\mathrm{cm})$ & $99.3 \pm 18.5$ & $94,1 \pm 18,5$ \\
\hline Course-Navette (paliers) & $3.0 \pm 1.5$ & $2.5 \pm 1.1$ \\
\hline zCF-ALPHA & $0.25 \pm 0.10$ & $0.19 \pm 0.10$ \\
\hline zmCF-ALPHA, $n(\%)$ & $16(40)$ & $39(61.9)$ \\
\hline zMCF-ALPHA, $n$ (\%) & $24(60.0)$ & $24(38.1)$ \\
\hline Actividad física & $3.3 \pm 1.8$ & $3.8 \pm 1.8$ \\
\hline No activo, n (\%) & 38 (95) & $49(77.8)$ \\
\hline Activo, n (\%) & $2(5)$ & $14(22.2)$ \\
\hline No activo/mzCF-ALPHA, $n$ (\%) & $29(40.0)$ & $45(50.8)$ \\
\hline No activo/MzCF-ALPHA, $n$ (\%) & $0(.00)$ & $18(11.1)$ \\
\hline Activo/zmCF-ALPHA, $n(\%)$ & $22(55.0)$ & $17(27.0)$ \\
\hline Activo/ zMCF-ALPHA, $n$ (\%) & $2(5.0)$ & $7(11.1)$ \\
\hline Conductual (0-18) & $15.5 \pm 2.2$ & $15.4 \pm 3.0$ \\
\hline Intelectual (0-17) & $12.7 \pm 2.7$ & $13.0 \pm 2.3$ \\
\hline Físico $(0-12)$ & $10.2 \pm 2.1$ & $9.9 \pm 2.1$ \\
\hline Ansiedad (0-12) & $8.6 \pm 2.5$ & $8.0 \pm 2.2$ \\
\hline Social (0-12) & $10.8 \pm 1.8$ & $10.7 \pm 2.0$ \\
\hline Satisfacción vital (0-9) & $7.8 \pm 1.5$ & $8.1 \pm 1.2$ \\
\hline Global $(0-80)$ & $66.8 \pm 9.6$ & $66.4 \pm 9.6$ \\
\hline
\end{tabular}

La tabla 3 muestra el análisis del autoconcepto en función del nivel de AF (No activo y activo). La prueba U de Mann-Whitney detectó que aquellos físicamenteactivos tuvieron mejores registros en las dimensiones conductual ( $p=.038)$, intelectual $(p=.001)$, falta de ansiedad $(p=.034)$ y global $(p=.007)$.

Por otro lado, cuando la muestra de participantes se categorizó según el nivel de CF (zCF-ALPHA), se observaron diferencias estadísticamente significativas en el autoconcepto (véase Tabla 4). Así, la prueba U de Mann-Whitney detectó que los escolares con zMCFALPHA mostraron un mejor autoconcepto físico $(p=.013)$ y social ( $p$ $=.003$ ). También se detectó una tendencia hacia la significación estadística en el autoconcepto global $(p=.085)$.

Como análisis complementario y con el propósito de verificar la relación combinada de la AF, la CF y el autoconcepto, se realizó un análisis mediante la prueba $\mathrm{H}$ de Kruskal-Wallis (véase tabla 5). Esta

\begin{tabular}{|c|c|c|c|c|c|c|c|}
\hline & $p$ valor \\
\hline Conductual (0-18) & \multicolumn{2}{|c|}{$15.2 \pm 2.8$} & \multirow{2}{*}{\multicolumn{2}{|c|}{$\begin{array}{l}16.6 \pm 1.6 \\
14.6 \pm 1.3\end{array}$}} & \multicolumn{2}{|c|}{-2.071} & .038 \\
\hline Intelectual (0-17) & \multirow{2}{*}{\multicolumn{2}{|c|}{$\begin{array}{c}12.5 \pm 2.5 \\
9.9 \pm 2.2\end{array}$}} & & & \multirow{2}{*}{\multicolumn{2}{|c|}{$\begin{array}{l}-3.419 \\
-0.600\end{array}$}} & .001 \\
\hline Físico $(0-12)$ & & & \multicolumn{2}{|c|}{$10.5 \pm 1.3$} & & & .549 \\
\hline Ansiedad $(0-12)$ & \multicolumn{2}{|c|}{$8.0 \pm 2.4$} & \multicolumn{2}{|c|}{$9.4 \pm 1.7$} & \multicolumn{2}{|c|}{-2.119} & .034 \\
\hline Social $(0-12)$ & \multicolumn{2}{|c|}{$10.6 \pm 2.0$} & \multicolumn{2}{|c|}{$11.4 \pm 1.2$} & \multicolumn{2}{|c|}{-1.626} & .104 \\
\hline Satisfacción vitz & \multicolumn{2}{|c|}{$7.9 \pm 1.4$} & \multicolumn{2}{|c|}{$8.3 \pm .9$} & \multicolumn{2}{|c|}{-0.433} & .665 \\
\hline Global $(0-80)$ & \multicolumn{2}{|c|}{$65.6 \pm 9.9$} & \multicolumn{2}{|c|}{$72.3 \pm 4.2$} & \multicolumn{2}{|c|}{-2.693} & .007 \\
\hline \multicolumn{8}{|c|}{$\begin{array}{l}\text { Nota. No activo }=\mathrm{X}<5 \text { horas, } \mathrm{y} \text { activo }=\mathrm{X}=5 \text { horas (Prochaska et al., 2001). Fuente: } \\
\text { elaboración propia. }\end{array}$} \\
\hline \multicolumn{8}{|c|}{$\begin{array}{l}\text { Tabla } 4 . \\
\text { Relación entre condición física y autoconcepto. }\end{array}$} \\
\hline & \multicolumn{2}{|c|}{$\begin{array}{c}\text { zmCF-ALPHA } \\
(\mathrm{n}=55)\end{array}$} & \multicolumn{2}{|c|}{$\begin{array}{l}\text { zMCF-ALPHA } \\
(\mathrm{n}=48)\end{array}$} & & $p$ valor \\
\hline Conductual (0-18) & \multicolumn{2}{|c|}{$15.3 \pm 3.2$} & \multicolumn{2}{|c|}{$15.6 \pm 2.0$} & \multicolumn{2}{|c|}{$\begin{array}{c}2 \\
-.373\end{array}$} & .710 \\
\hline Intele & \multicolumn{2}{|c|}{$12.7 \pm 2.5$} & $13.0 \pm 2$. & & -.98 & & .325 \\
\hline Físico & & $5 \pm 2.3$ & $10.5 \pm 1.7$ & & -2.4 & & .013 \\
\hline Ansied & & $8 \pm 2.6$ & 8.7 & & -1.5 & & 132 \\
\hline Social & & $4 \pm 2.0$ & 11.2 & & -2.9 & & .003 \\
\hline Satisfacción vital (0-9) & & $8 \pm 1.5$ & $8.2 \pm 1.1$ & & -1.0 & & .314 \\
\hline Global $(0-80)$ & 64.8 & $8 \pm 10.9$ & $68.7 \pm 7.3$ & & -1.7 & & .085 \\
\hline $\begin{array}{l}\text { Nota. CF-ALPHA = c } \\
\text { MzCF-ALPHA = may } \\
\text { elaboración propia. }\end{array}$ & ondición físi & $\begin{array}{l}\text { ica ALPHA } \\
\mathrm{P}_{50} \text { ) (Rosa- }\end{array}$ & ZzCF-ALPF & & & & $\begin{array}{l}\left.X<P_{50}\right), \\
\text { ). Fuente: }\end{array}$ \\
\hline $\begin{array}{l}\text { Tabla } 5 . \\
\text { Relación de la actividad }\end{array}$ & a & ondici & 40 & & & & \\
\hline & $\mathrm{A}(\mathrm{n}=48)$ & $\mathrm{B}(\mathrm{n}=7)$ & $\mathrm{C}(\mathrm{n}=38)$ & $\mathrm{D}(\mathrm{n}=$ & =9) & $\mathrm{Z}$ & $p$ valor \\
\hline Condt & $15.1 \pm 3.3$ & $16.6 \pm 2.3$ & $15.4 \pm 2.1$ & & & 4.555 & .207 \\
\hline Intelec & $12.4 \pm 2.5$ & $14.4 \pm 1.7$ & $12.6 \pm 2.5$ & & & 12.097 & .007 \\
\hline Físico & $9.5 \pm 2.4$ & $9.9 \pm 1.1$ & $10.4 \pm 1.8$ & $11.0=$ & \pm 1.2 & 6.506 & .089 \\
\hline -12) & $7.6 \pm 2.7$ & $9.1 \pm 1.8$ & $8.5 \pm 1.9$ & & & 6.220 & .101 \\
\hline & $10.4 \pm 2.0$ & $10.7 \pm 1.6$ & $10.9 \pm 2.0$ & 12.0 & \pm .0 & 11.382 & .010 \\
\hline Satisfacción vital (0-9) & $7.8 \pm 1.5$ & $8.0 \pm 1.0$ & $8.1 \pm 1.2$ & & & 1.493 & .684 \\
\hline Global (0-80) & $64.0 \pm 11.4$ & $69.9 \pm 5.2$ & $67.2 \pm 7.6$ & $74.1=$ & \pm 1.9 & 10.651 & .014 \\
\hline
\end{tabular}

ALPHA; $\mathrm{D}=$ activo/zMCF-AL PHA. Fuente: elaboración propia. 
prueba detectó que los escolares (no activos y activos) con zMCFALPHA tenían en general un mejor autoconcepto que sus pares homólogos con zmCF-ALPHA. Concretamente, los escolares activos y con mayor CF (activo/zMCF-ALPHA) tuvieron un mejor autoconcepto intelectual $(p=.007)$, social $(p=.010)$, y global $(p=$ $.010)$, con respecto al resto de los grupos. Ademásse observó una tendencia hacia la significación estadística en el autoconcepto físico ( $p=$ .089).

\section{Discusión}

Este estudio trató de clarificar en 40 niños y 63 niñas de ocho a 12 años, si los niveles de AF y CF se relacionaban con el autoconcepto multidimensional, empleando para ello estadística no paramétrica, y representa uno de los escasos análisis realizados en niños de enseñanza primaria.

El estudio de los factores que pueden tener cierta influencia en la configuración positiva del autoconcepto es de gran relevancia en la edad escolar, ya que representa uno de los indicadores más relevantes para un adecuado funcionamiento físico, cognitivo, comportamental, afectivo y social (Madariaga \& Goñi, 2009). Un bajo autoconcepto puede influir negativamente en la noción que el niño tiene sobre su potencial humano en un determinado rango de situaciones, provocando que no se sienta bien consigo mismo y con sus capacidades, teniendo más probabilidades de padecer desórdenes psicosociales como ansiedad, depresión, síntomas psicopatológicos o baja autoestima (Gálvez et al., 2015c; Garaigordobil, Pérez y Mozaz, 2008; García, 2013).

Los análisis estadísticos realizados satisfacen el objetivo de este trabajo, indicando la existencia de una relación positiva entre la AF, la CF y el autoconcepto multidimensional. De esta manera, aquellos físicamente activos se percibieron mejor en las dimensiones conductual ( $p=$ $.038)$, intelectual $(p=.001)$, falta de ansiedad $(p=.034)$ y global $(p=$ .007), que sus semejantes no activos (véase tabla 3 ).

Estos resultados se encuentran en la misma línea de otros que indican una asociación positiva entre la AF y la falta de ansiedad (Petty et al., 2009) ( $n=207,7-11$ años), la AF y el autoconcepto académico (Kyle et al., 2016) ( $n=63,10-12$ años), la AF y el autoconcepto global (García-Cantó y García-López, 2011) (n = 207, 10-12 años).

Las conclusiones de estos estudios ponen de relieve que el rendimiento de los niños en distintos ámbitos puede verse influido, en buena parte, por la consistencia de la asociación de las conductas intencionales y el autoconcepto multidimensional, siendo una de ellas la realización habitual de AF en el ámbito académico, debido a que las experiencias a nivel educativo pueden ser muy positivas para el alumnado, pudiéndose ver esto reflejado en patrones generales de conducta y aprendizaje más equilibrados y constructivos.

Es lógico pensar desde la concepción multidimensional del autoconcepto de Shavelson et al. (1976), que la relación entre la AF y el autoconcepto se encuentra mediatizada por uno de sus dominios, el autoconcepto físico. En este trabajo no se hallaron diferencias estadísticamente significativas en el autoconcepto físico, en contraposición a lo hallado por Mendo-Lázaro et al. (2017) (n = 944, 9-12 años), que indican que los niños practicantes de AF y deportivas organizadas, además de percibirse mejor físicamente, poseen un mejor control sobre las situaciones y las emociones, dado que algunos de los factores que caracterizan la práctica de los deportes organizados, como la competitividad, la amistad y el compromiso, podrían materializarse en unos valores más elevados del autoconcepto físico y emocional; en línea también con el Modelo de Ejercicio y Autoestima de Sonströem et al. (1994), que señalan que la mejora de la autoeficacia física conduce a una mayor autoeficacia percibida que realzaría la autopercepción general mejorando a su vez la autoestima. Esto lo podemos constatar en cierta medida, ya que los niños activos mostraron valores absolutos más elevados en todas las dimensiones del autoconcepto, incluida la física, repercutiendo en una mejor valoración global del mismo (no activos, $65.6 \pm 9.9$ vs. activos, $72.3 \pm 4.2$ ).

No obstante, Goñi y Zulaika (2000) en un estudio (n = 153, 10-11 años) afirman que a esta edad aún no se ha consolidado el autoconcepto mostrando la AF una escasa influencia en la configuración del mismo; a diferencia de lo aportado por Planinšec \& Fošnariè (2005) (n = 364, 67 años) y Moreno et al. (2008) ( $n=578,9-23$ años), que hallaron una relación estadísticamente significativa entre AF y autoconcepto, aunque no pudieron demostrar la dirección de esta relación. En este sentido, Zulaika (2002) ( $n=153,10$ años) señala que un autoconcepto positivo está en la base del buen funcionamiento personal y social; de él depende, en buena medida, la satisfacción personal, el sentirse bien consigo mismo, en suma, la autorrealización en distintos ámbitos. Por ello, un elevado autoconcepto y un sentido de autoeficacia podrían tener una influencia sobre la mejora del rendimiento físico y viceversa (SánchezAlcaraz \& Gómez-Mármol, 2014; Hortigüela-Alcalá, Pérez-Pueyo, \& Calderón-Luquín, 2016).

En este trabajo, se encontró que los niños con mayor nivel de CF (zMCF-ALPHA) mostraron un mejor autoconcepto físico ( $p=.013$ ), social ( $p=.003)$, y global $(p=.085)$, que sus pares homólogos con menor nivel (véase tabla 4). Hallazgos similares se obtuvieron en otros estudios donde se analizó la relación entre CF y autoconcepto multidimensional (Rodríguez-García et al., 2014), fuerza muscular global y autoconcepto multidimensional (Rodríguez-García et al., 2015) y CF y autoconcepto físico global (Sánchez-Alcaraz y Gómez-Mármol, 2014; Mayorga et al., 2012).

Según García (2013) la mejora de las funciones (biológicas y psicológicas) que determinan el estado de $\mathrm{CF}$, puede contribuir a una mayor habilidad de los niños para solucionar problemas con los iguales, a la aceptación social y a la adaptación al medio, debido a que suelen presentar una mayor participación en AF y deportivas, en coherencia con lo planteado por Mendo-Lázaro et al. (2017).

En relación a esto, aunque no pueden establecerse relaciones de causalidad entre las variables analizadas, estos estudios coinciden en revelar a la $\mathrm{AF}$ como una estrategia para mejorar el estado de $\mathrm{CF}$, que afecta positivamente a la salud física y al bienestar mental; aportando evidencia empírica en línea de propuestas como la de Rodríguez-García et al. (2014) que subrayan la importancia adquirida por la CF durante los últimos años en el análisis del autoconcepto; o la de Moore et al. (2011) que contemplan la AF como una conducta intencional que puede incidir positivamente en los niveles de autoconcepto en niños y jóvenes. Sin embargo, Guillén \& Ramírez (2011) en su estudio ( $=75$, 10 12 años) concluyen que en edad preadolescente, diversos indicadores de la CF no influyen sobre el autoconcepto global, y se relacionan débilmente con alguna de sus dimensiones como la agilidad con la intelectual, y la fuerza resistencia abdominal con la conductual, y la felicidad-satisfacción vital.

En este estudio, los niños físicamente activos y con mayor CF (activo/zMCF-ALPHA) tuvieron un mejor autoconcepto intelectual ( $p=.007)$, social $(p=.010)$ y global $(p=.010)$, en comparación con el resto de grupos. Asimismo, se observó una tendencia hacia la significación estadística en el autoconcepto físico ( $p=.089$ ).

En la preadolescencia, el niño comienza a notar importantes cambios corporales, sexuales, emocionales y sociales, además de cerebrales (Mendo-Lázaro et al., 2017), que se traducen en beneficios obtenidos de poseer un mayor nivel de AF y CF; reflejándose a su vez en un mayor sentido de responsabilidad, autocontrol, autonomía y una mayor competencia para cualquier tipo de actividad (Ramírez \& Vega, 2018; García et al., 2013).

Los resultados de este estudio se muestran coherentes, en parte, con lo reportado por Vedul Kjelsås et al. (2012) ( $n=67,10-13$ años), los cuales encontraron una consistente relación entre la competencia motriz, la CF, y la autopercepción global, señalando a estos factores como esenciales para facilitar la participación y la adherencia hacia la AF en edades tempranas (Zurita-Ortega y cols., 2018). En este sentido, GarcíaSánchez et al. (2013) a pesar de encontrar relaciones estadísticamente significativas solo con las dimensiones física y social, sugieren que estar en buena CF contrarresta el bajo autoconcepto asociado al sobrepeso/ obesidad, alcanzando los jóvenes que mejoran su capacidad aeróbica niveles similares de autoconcepto que aquellos con normopeso. Una 
estrategia para mejorar la CF y con ello el autoconcepto multidimensional podría ser la realización adecuada de AF (véase figura 1).

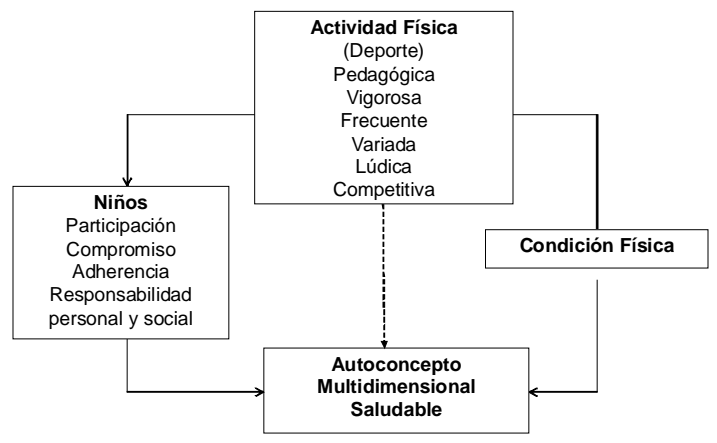

Figura 1.

Modelo explicativo acerca de cómo podría influir la actividad física y la condición física sobre el autoconcepto. Fuente: elaboración propia.

Gálvez et al (2015c) indican la importancia de difundir los resultados de estas investigaciones entre la comunidad educativa con la finalidad de mejorar el autoconcepto multidimensional mediante la concepción de la AF y el deporte como instrumentos pedagógicos; además de complementarlos con otros apoyos de índole cognitiva y emocional, debido a su estrecha conexión con el aprendizaje escolar (Fraile \& Catalina, 2013; Navarro-Paton, Ferreira, \& García, 2018).

Siendo conscientes de las limitaciones de un estudio transversal y con una muestra no representativa de la población preadolescente, los resultados de este trabajo ponen de manifiesto una asociación entre la AF, la CF y el autoconcepto multidimensional. Siguiendo los modelos explicativos del autoconcepto actualmente aceptados, en la determinación de las autopercepciones de las personas no solo intervendrían el medio sociocultural y las conductas intencionales, dentro de las cuáles se incluye la AF, sino que también habría que tener en cuenta otros factores como la predisposición genéticaque en este estudio no se ha tenido en cuenta. Sin embargo, el hecho de que esta investigación se haya llevado a cabo por personas implicadas en la docencia y en contacto directo con la salud y la AF ha supuesto una ventaja pues las aportaciones se ofrecen desde una perspectiva cercana a los niños, y desde las necesidades detectadas por la experiencia docente (Navas \& Soriano, 2016).

En conclusión, se puede sugerir que tanto laAF como la CF pueden ser elementos diferenciadores del autoconcepto multidimensional en niños españoles de ocho a 12 años. Ser físicamente activo y tener un mayor nivel de CF podría predecir un autoconcepto más positivo en las etapas infantil y adolescente. Por todo esto, es necesario plantear como objetivos evaluables para el profesorado de educación física el incremento de las oportunidades de AF y la mejora del estado de CF de su alumnado en la enseñanza obligatoria.

\section{Referencias}

Bufford, R. K. (1986). Social foundations of thought and action-a social cognitive theory-bandura, a. Journal of Psychology and Theology, 14(4), 341-342.

Calfas, K. J., Long, B. J., Sallis, J. F., Wooten, W. J., Pratt, M., \& Patrick, K. (1996).A controlled trial of physician counseling to promote the adoption of physical activity. Preventive medicine, 25(3), 225-233.

Cardenal, V., \& Fierro, A. (2003). Componentes y correlatos del autoconcepto en la escala de Piers-Harris. Estudios de Psicología 24, 101-111.

Casajus, J. A., Ortega, F. B., Vicente-Rodriguez, G., Leiva, M. T., Moreno, L. A., \& Ara, I. (2012). Physical fitness, fat distribution and health in school-age children (7 to 12 years). Revista Internacional de Medicina y Ciencias de la Actividad Fisica y del Deporte, 12(47), 523-537.

Castro-Pinero, J., Ortega, F. B., Artero, E. G., Girela-Rejón, M. J., Mora, J., Sjostrom, M., \& Ruiz, J. R. (2010). Assessing muscular strength in youth: usefulness of standing long jump as a general index of muscular fitness. Journal of Strength and Conditioning Research, 24(7), 1810-
1817.

Department of Health, Physical Activity, Health Improvement and Prevention (2004). At least five a week: Evidence on the impact of physical activity and its relationship to health. A report from the Chief Medical Officer. London: Department of Health.

España-Romero, V., Ortega, F. B., Vicente-Rodríguez, G., Artero, E. G., Rey, J. P., \& Ruiz, J. R. (2010). Elbow Position Affects Handgrip Strength in Adolescents: Validity and Reliability of Jamar, Dynex, and Tkk Dynamometers. Journal of Strength and Conditioning Research, 24(1), 272-277.

Flores, P. E., Medrano, L. A., \& Victoria, L. M. (2014). Estados de Ánimo y Juicios de Autoconcepto en Universitarios: Análisis desde un abordaje basado en Redes Semánticas Naturales. Interamerican Journal of Psychology, 48(3).

Fraile, A., \& Catalina, J. (2013). Diferencias en autoconcepto físico en escolares de primaria y secundaria. Lúdicapedagógica, 2(18), 93102.

Gallego, F. L., Sánchez, A. J. L., Vacas, N. E., \& Zagalaz, J. C. (2016). Influencia del género, la edad y el nivel de actividad física en la condición física de alumnos de educación primaria. Revisión Bibliográfica. Retos: nuevas tendencias en educación física, deporte y recreación, (29), 129-133.

Gálvez, A., Rodríguez-García, P. L., Rosa, A., García-Cantó, E., PérezSoto, J. J., Tárraga, M.L., Tárraga, P.J. (2015c). Relación entre el estatus de peso corporal y el autoconcepto en escolares. Nutrición Hospitalaria, 31(2), 723-729.

Gálvez, A., Rodríguez-García, P.L., García-Cantó, E., Rosa, A., PérezSoto, J.J., Tárraga-Marcos, M.L., Tárraga-Marcos, P.J. (2015a). Capacidad aeróbica y calidad de vida en escolares de 8 a 12 años. Clínica e Investigación en Arteriosclerosis, 27(5), 239-25.

Gálvez, A., Rodríguez-García, P.L., Rosa, A., García-Cantó, E., PérezSoto, J.J., Tárraga, M.L., Tárraga, P.J. (2015b). Nivel de condición física y su relación con el estatus de peso corporal en escolares. Nutrición Hospitalaria, 31(1), 393-400.

Gálvez, A., Rodríguez-García, P.L., Rosa, A., García-Cantó, E., PérezSoto, J.J., Tárraga, P.J., Tárraga, M.L. (2016). Capacidad aeróbica, estado de peso y autoconcepto en escolares de primaria. Clínica e Investigación en Arteriosclerosis, 28(1), 1-8.

Garaigordobil, M., Perez, J. I., \& Mozaz, M. (2008). Self-concept, selfesteem and psychopathological symptoms. Psicothema, 20(1), 114123.

García, A.R. (2013). La educación emocional, el autoconcepto, la autoestima y su importancia en la infancia. Edetania: estudios y propuestas socio-educativas, 44, 241-258.

García-Cantó, E., \& García-López, A. (2011). El autoconcepto y la práctica de actividad física en Primaria. Trances, Revista de Transmisión del Conocimiento Educativo y de la Salud, 3(2), 187-200.

García-Sánchez, A., Burgueño-Menjibar, R., López-Blanco, D. y Ortega, F.B. (2013). Condición física, adiposidad y autoconcepto en adolescentes. Estudio piloto. Revista de Psicología del Deporte, 22(2), 453-461.

Goñi, A., \& Zulaica, L.M. (2000). La participación en el deporte escolar y el autoconcepto en escolares de 10 a 11 años de la provincia de Guipúzcoa. Apunts, 59, 6-10.

Goñi, E., \& Fernández, A. (2008). Los dominios social y personal del autoconcepto. Revista de Psicodidáctica, 12(2), 179-194.

Guillén, F., \& Ramírez, F. (2011). Relación entre el autoconcepto y la condición física en alumnos del Tercer Ciclo de Primaria. Revista de Psicología del Deporte, 20(1), 45-49.

Hortigüela-Alcalá, D., Pérez-Pueyo, A., \& Calderón-Luquín, A. (2016). Efecto del modelo de enseñanza sobre el autoconcepto físico del alumnado en educación física. Retos, Nuevas tendencias en Educación Física, Deporte y Recreación, 30, 76-81

Kyle, T. L., Hernández-Mendo, A., Reigal-Garrido, R. E., \& MoralesSánchez, V. (2016). Efectos de la actividad física en el autoconcepto y la autoeficacia en preadolescentes. Retos. Nuevas tendencias en Educación Física, Deporte y Recreación, 29, 61-65.

Lèger, L.A., Mercier, D., Gadoury, C., \& Lambert, J. (1988). Themultistage 20 meter shuttle run test for aerobic fitness. Journal of Sports Sciences, 6(2), 93-101. 
Madariaga, J., \& Goñi, A. (2009). El desarrollo psicosocial. Revista de Psicodidáctica, 14(1), 93-118.

Manual de Instrucciones de la Batería ALPHA-Fitness: Test de campo para la evaluación de la condición física relacionada con la salud en niños y adolescentes. [Acceso: 15 de septiembre de 2016]. Disponible en: https://sites. google.com/site/alphaprojectphysicalactivity/ alphapublic-documents/alpha-fit/assessing-fitness-in-children.

Martínez-Baena, A., Mayorga-Vega, D., \& Viciana, J. (2018). Factores predictores de la actividad física en escolares españoles de acuerdo a su estado de peso. Retos. Nuevas tendencias en Educación Física, Deporte y Recreación, 33, 74-80.

Mayorga, D., Viciana, J., \& Cocca, A. (2012). Relationship between physical self-concept and health-related physical fitness in Spanish schoolchildren. Procedia-Social and Behavioral Sciences, 69, 659668.

Mendo-Lázaro, S., Polo-del-Río, M. I., Amado-Alonso, D., Iglesias-Gallego, D., \& León-del-Barco, B. (2017). Self-concept in childhood: the role of body image and sport practice. Frontiers in Psychology, 8.

Mitchell, N.G., Moore, J.B., Bibeau, W.S., \& Rudasill, K.M. (2012). Cardiovascular fitness moderates the relations between estimates of obesity and physical self-perceptions in rural elementary school students. Journal of Physical Activity and Health, 9(2), 288-294

Moore, J. B., Mitchell, N. G., Bibeau, W. S., \& Bartholomew, J. B. (2011) Effects of a 12-week resistance exercise program on physical selfperceptions in college students. Research Quarterly Exercise Sport, 82(2), 291-301.

Moreno, J. A, Cervelló, E., \& Moreno, R. (2008). Importancia de la práctica físico-deportiva y del género en el autoconcepto físico de los 9 a los 23 años. International Journal of Clinical and Health Psychology, 8(1), 171-183.

Navarro-Paton, R., Ferreira, B. R., \& García, M. G. (2018). Incidencia de los juegos cooperativos en el autoconcepto físico de escolares de educación primaria. Retos, nuevas tendencias en educación física, deporte y recreación (34), 14-18.

Navas, L., \& Soriano, J. A. (2016). Análisis de los motivos para practicar o no actividades físicas extracurriculares y su relación con el autoconcepto físico en estudiantes chilenos. Revista iberoamericana de psicología del ejercicio y el deporte Vol. 11 n 1 pp. 69-76

Patrick, K., Sallis, J. F., Prochaska, J. J., Lydston, D. D., Calfas, K. J., Zabinski, M. F... Brown, D. R. (2001). A multicomponent program for nutrition and physical activity change in primary care: PACE + for adolescents. Archive Pediatric Adolescent Medicine, 155, 940-6.

Petty, K. H., Davis, C. L., Tkacz, J., Young-Hyman, D., \& Waller, J. L. (2009). Exercise effects on depressive symptoms and self-worth in overweight children: arandomized controlled trial. Journal of Pediatric Psychology, 34(9), 929-939.

Piers, E. V., \& Herzberg, D. S. (2002).Manual for the Piers-Harris Children's Self-concept Scale (2nd edn). Los Ángeles, CA: Western Psychological Services.

Planinšec, J., \& Fošnariè, S. (2005). Relationship of perceived physical self-concept and physical activity level and sex among young children. Perceptual and motor skills, 100(2), 349-353.

Prochaska, J.J., Sallis, J.F., \& Long, B. (2001). A physical activity screening measure for use with adolescents in primary care. Arch Pediatr Adolesc Med, 155, 554-9.

Ramírez, J. V., \& Vega, D. M. (2018). The three-axes model of planning in physical education. Retos: nuevas tendencias en educación física, deporte y recreación, (33), 313-319.

Reigal, R., Videra, A., Parra, J. L., \& Juárez, R. (2012). Actividad físico deportiva, autoconcepto físico y bienestar psicológico en la adolescencia. Retos. Nuevas tendencias en Educación Física, Deporte y Recreación, 22, 19-23.

Reynaga-Estrada, P., Arévalo Vázquez, E. I., Verdesoto Gáleas, A. M., Jiménez Ortega, I. M., Preciado Serrano, M. L., \& Morales Acosta, J. J. (2016). Beneficios psicológicos de la actividad física en el trabajo de un centro educativo. Retos, nuevas tendencias en Educación Física, Deporte y Recreación, 30, 203-206.

Rodríguez-García, P.L., Gálvez, A., García-Cantó, E., Pérez-Soto, J.J., Rosa, A., Tárraga... Tárraga, P.L. (2015). Relationship between the Self-Concept and Muscular Strength in Southern Spanish Children. $J$
Psychol Psychother 5, 222.

Rodríguez-García, P.L., Tárraga, L., Rosa, A., García-Cantó, E., PérezSoto, J.J., Gálvez, A., Tárraga, P. (2014). Physical Fitness Level and Its Relationship with Self-Concept in School Children. Psychology, 5, 2009-2017.

Rosa-Guillamón, A., \& García-Cantó, E. (2016). Relationship between physical fitness and mental health in primary school children. Rev Ib CC Act Fís Dep, 5(2), 31-42.

Rosa-Guillamón, A., García-Cantó, E., Rodríguez-García, P. L., \& PérezSoto, J. J. (2016). Condición física y calidad de vida en escolares de 8 a 12 años. Revista de la Facultad de Medicina, 65(1), 37-42.

Rosa-Guillamón, A., García-Cantó, E., Rodríguez-García, P. L., PérezSoto, J.J., Tárraga-Marcos, M.L., Tárraga-López, P.J. (2017). Actividad física, condición física y calidad de la dieta en escolares de 8 a 12 años. Nutr Hosp, 34(6), 1292-1298.

Ruiz, J. R., España Romero, V., Castro Piñero, J., Artero, E. G., Ortega, F. B., Cuenca, M., Castillo, M. J. (2011). Batería ALPHA-Fitness: test de campo para la evaluación de la condición física relacionada con la salud en niños y adolescentes, Nutr Hosp, 26(6), 1210-1214.

Sánchez-Alcaraz, B. J., \& Gómez-Mármol, A. (2014). Physical self-concept in a sample of primary students and its relationship with gender and out of school sport practice. E-balonmano. com: Revista de Ciencias del Deporte, 10(2), 113-120.

Shavelson, J., Hubner, J.J., \& Stanton, G.C. (1976). Self-concept: validation of construct interpretations. Review of Educational Research, 46, 407-442.

Sonströem, R.J. (1998). Physical self-concept: assessment and external validity. Exercise and Sport Sciences Reviews, 26, 133-164.

Sonströem, R.J., Harlow, L.L., \& Josephs, L. (1994). Exercise and selfesteem: Validity of modelexpansion and exercise associations. Journal of Sport and Exercise Psychology, 16, 29-42.

Strauss, R. S., Rodzilsky, D., Burack, G, \& Colin, M. (2001). Psychosocial correlates of physical activity in healthy children. Archives of Pediatrics \& Adolescent Medicine, 155(8), 897-902.

Thomas, S., Reading, J., \& Shephard, R. J.(1992).Revision of the physicalactivity readiness questionnaire (PAR-Q).Canadian Journal of Sport Sciences-Revue Canadienne Des Sciences Du Sport, 17(4), 338345.

Van den Berghe, L., Vanstenkiste, M., Cardon, G., Kirk, D., \& Haerens, L. (2014). Research on self determination in physicaleducation: keyfindings and proposal for future research. Physical Education and Sport Pedagogy, 19(1), 97-121.

Vedul Kjelsås, V., Sigmundsson, H., Stensdotter, A. K., \& Haga, M. (2012). The relationshipbetweenmotorcompetence, physical fitness and self perception in children. Child: care, health and development, 38(3), 394-402.

Vicente-Rodríguez, G, Rey-López, J. P., Mesana, M. I., Poortvliet, E., Ortega, F. B., Polito, A.,... Moreno, L. A. (2012). Reliability and intermethod agreement for body fat assessment among two field and two laboratory methods in adolescents. Obesity, 20(1), 221-228.

Weiss, M. R. (2000). Motivating kids in physical activity. President's Council on Physical Fitness and Sports Research Digest, 3(11), 1-8.

Zabala, A. F., \& Palacios, E. G. (2008). El autoconcepto infantil: una revisión necesaria. International Journal of Developmental and Educational Psychology, 2(1), 13-22.

Zagalaz-Sánchez, M. L., Castro-López, R., Valdivida-Moral, P., \& CachónZagalaz, J. (2018). Relacion entre autoconcepto físico, ansiedad y personalidad manifestada en usuarios de gimnasios Retos: nuevas tendencias en educación física, deporte y recreación (32), 53-57.

Zulaika, L.M. (2002). Educación física y autoconcepto: análisis relacionales y eficiencia de un programa de intervención para la mejora del autoconcepto físico. Apunts: Educación física y deportes, 67, 114.

Zurita-Ortega, F., Ubago-Jiménez, J. L., Puertas-Molero, P., GonzálezValero, G, Castro-Sánchez, M., \& Chacón-Cuberos, R. (2018). Niveles de actividad física en alumnado de Educación Primaria de la provincia de Granada Retos: nuevas tendencias en educación física, deporte y recreación (34), 218-221. 